\title{
Actitudes hacia la inmigración de los estudiantes de psicología chilenos: análisis diferenciales
}

\section{Attitudes toward immigration in chileans students of psychology: differential analysis}

\author{
Leandro Navas ${ }^{1}$ y Alejandra Sánchez \\ Universidad de Alicante, España
}

(Recepción: Octubre 2009 - Aceptación: Diciembre 2009)

\begin{abstract}
Resumen. El objetivo del estudio fue analizar las actitudes hacia la inmigración de un grupo de estudiantes de psicología chilenos. Participaron 117 sujetos con edades comprendidas entre los 18 y 33 años. Se aplicó la Escala de Actitudes hacia la Inmigración (León, Mira y Gómez, 2007). Los resultados indicaron que el instrumento seleccionado presenta coeficientes de fiabilidad aceptables y que las actitudes de los sujetos muestran una tendencia favorable, excepto en la variable distancia social positiva. No obstante, un número importante de sujetos mostró una actitud intermedia, la cual se podría relacionar con un prejuicio sutil. Existen diferencias en las actitudes que favorecen a las mujeres y a los individuos que se han relacionado con personas inmigrantes.

Palabras clave: Actitud, inmigración, inmigrante, prejuicio.
\end{abstract}

\begin{abstract}
The aim of the study was to analyzed attitudes toward immigration in a group chileans student of psychology. 117 individuals aged between 17 and 33 were involved. We administred the Attitudes toward Immigration Scale (León, Mira \& Gómez, 2007). Results indicated that the selected instrument presents coefficients of reliability acceptable and that the attitudes of subjects show a favorable trend, except for the variable positive social distance. However, a significant number of subjects showed an intermediate attitude, which could be related to subtle prejudice. There are differences in attitudes that favor women and individuals who have been associated with immigrants.
\end{abstract}

Key words: Attitude, immigration, immigrant, prejudice.

1 Correspondencia: Leandro Navas Martínez. Universidad de Alicante, Departamento de Psicología Evolutiva y Didáctica. Apartado de correos 99. CP. 03080, Alicante, España. E-Mail: Leandro.Navas@ua.es Teléfono: 965903400 (Extensión 2913) Fax: 965909886 


\section{Introducción}

Chile, desde sus inicios, se ha construido como nación con las aportaciones de las diversas culturas colonizadoras (e.g., española, inglesa y alemana) y de las diferentes etnias originarias (e.g., mapuches, atacameños y aymaras), lo que conlleva que, a lo largo del territorio nacional, se puedan apreciar diversos grupos culturales con costumbres y prácticas propias. Sin embargo, en la última década, la presencia masiva de inmigrantes obliga a reflexionar sobre las relaciones entre las minorías que llegan del extranjero y la sociedad de acogida.

El último censo de población realizado durante el año 2002, señala que el flujo migratorio hacia Chile aumentó en un 75\% durante el periodo comprendido entre 1992 y 2002. Por otra parte, de acuerdo con la información disponible en el Ministerio del Interior de Chile, alrededor del 2\% de la población chilena está constituida por extranjeros. En la actualidad, los inmigrantes que llegan a Chile proceden principalmente de países vecinos, tales como Perú, Argentina, Ecuador y Bolivia, pero también de otros continentes, todos ellos con otras costumbres, otras tradiciones, otro color de piel y otras religiones. En consecuencia, es razonable pensar que este país avanza hacia una sociedad pluricultural.

No obstante, Chile ha tenido un plazo relativamente breve para aceptar y responder eficazmente a su creciente diversidad cultural, ya que la legislación vigente resulta inadecuada para hacer frente a los desafíos que la inmigración genera. Según el último informe sobre los derechos humanos en Chile (Quesille, 2007), los inmigrantes constituyen uno de los grupos minoritarios cuyos derechos fundamentales se encuentran en el más alto nivel de vulnerabilidad. Además, a estas dificultades se les agregan las respuestas sociales xenófobas y discriminatorias de la población autóctona. En tales ideas encuentra justificación el estudio de las actitudes negativas que, a pesar de las iniciativas impulsadas por algunas organizaciones sociales y del rechazo público que ha realizado el gobierno, se han mantenido y empeorado en la medida en la que aumenta el número de inmigrantes en el país andino.

Teniendo en cuenta la basta experiencia de aquellos países con larga trayectoria de inmigración y el cambio en el clima cultural que esto ha generado, es poco probable que los países que recientemente están conociendo una creciente inmigración, como es el caso de Chile, atraviesen una primera etapa de hostilidad abierta, más aún si esos sentimientos son combatidos con decisión por la opinión pública.

Tanto Dovidio y Gaertner (1991, 1998) como Pettigrew y Meertens (1995) plantean que, en los últimos años, se ha producido una progresiva y sensible reducción del prejuicio manifiesto y de la aversión explícita con respecto a las minorías culturales. No obstante, sostienen que la hostilidad hacia el exogrupo sobrevive de forma enmascarada y sutil, adaptada para convivir con los valores universalmente aceptados de tolerancia, igualdad y dignidad humana. Es decir, actualmente, el prejuicio y la discriminación cultural se manifiestan, en alta medida, de modo implícito y poco reconocible. Por ejemplo, se puede exteriorizar a través de la oposición, con diversas motivaciones, aparentemente, no racistas, a iniciativas públicas dirigidas a favorecer a las minorías o por el distanciamiento, producido por la contradicción entre los propios valores igualitarios y un antiguo y enraizado sentimiento de hostilidad frente a los miembros del exogrupo (Katz \& Hass, 1988).

En la mayoría de los modelos teóricos sobre las actitudes hacia la inmigración está implícita la idea de que las actitudes negativas son una forma de prejuicio porque se evalúa a las personas no como individuos, sino sobre la base de su pertenencia a un grupo (Tajfel, 1981; Turner, Hogg, Oakes, Reicher \& Wetherell, 1987). Según Morales y Huici (1999), en el momento de la interacción, se desestiman las características individuales del sujeto y se le percibe teniendo en cuenta el hecho de que pertenece a una categoría social.

Existe un considerable debate teórico sobre si el prejuicio hacia los inmigrantes se deriva de factores económicos o de factores ideológicos (O’Rourke \& Sinnott, 2006), es decir, si procede de los intereses o de la ideología de las personas. Los argumentos sobre los intereses suelen centrarse 
en el vínculo existente entre el mercado laboral y la lucha contra la inmigración. Dado que los inmigrantes son reclutados, a menudo, para atender la escasez de mano de obra en el mercado laboral, la inmigración supone una competencia para la población autóctona (Olzak, 1992). Incluso, se ha argumentado que no es necesario que esta competencia sea real, sólo basta con que se perciba (Raijman, Semyonov \& Schmidt, 2003; Semyonov, Raijman, Yom Tov \& Schmidt, 2004). Las explicaciones basadas en la ideología se centran en un conjunto amplio de creencias sobre la superioridad de una cultura sobre otra. Desde esta perspectiva, el deseo de frenar la inmigración es parte de una mayor tendencia hacia el racismo y la xenofobia (Dustmann y Preston, 2004; Gutiérrez, 1995).

Tratando de responder a la cuestión acerca de qué actitud manifiesta la población hacia la inmigración, un estudio realizado por Simon y Sikich (2007) analizó las actitudes que manifestaban ciudadanos de distintos países (Estados Unidos, Canadá, Australia, Gran Bretaña, Alemania y Japón) frente al fenómeno de la inmigración. Los resultados señalaron que, en los distintos contextos, la actitud general de los individuos era negativa. Particularmente, la idea de enriquecimiento cultural fue, altamente, apoyada por los autóctonos, pero cuando este enriquecimiento supone un efecto de "mejora” para el país de acogida, los porcentajes de respuestas favorables caen alrededor del $20 \%$. Por otra parte, señalan que, con el transcurso del tiempo, el beneficio económico que genera la mano de obra inmigrante ha ido disminuyendo en la percepción de los encuestados. Además, la gran mayoría de los sujetos, excepto los de Estados Unidos y Canadá, asociaron el incremento de la delincuencia con los inmigrantes y, aproximadamente, la mitad de los individuos opinaron que los inmigrantes quitan puestos de trabajo a los autóctonos. Con estas percepciones, era de esperar que, la gran mayoría, excepto los canadienses, no deseen acoger un mayor número de inmigrantes en su país. Otro aspecto a destacar de los resultados es que, en la mayoría de los países y en los diversos temas consultados, el porcentaje de sujetos que manifestó una actitud intermedia (ni a favor ni en contra de la inmigración) rondó el 30\%, es decir, no existe una expresión explicita de actitudes negativas pero tampoco de actitudes positivas, situación que podría ser interpretada como una manifestación de la hostilidad encubierta o del prejuicio sutil.

Dentro de las variables que introducen diferencias en las actitudes hacia la inmigración, tradicionalmente, se mencionan el sexo, la edad, el nivel educativo y la competencia profesional de las personas (Gang, Rivera-Batiz \& Yun, 2001; Haubert \& Fusell, 2006; Molero, Navas \& Morales, 2001; Morales, 2003; Palmer, 1996; Pantoja, 2006; Tucci, 2005). Otras investigaciones han encontrado apoyo empírico para nuevas variables, entre las cuales se mencionan la experiencia de relación con personas inmigrantes (Pettigrew \& Tropp, 2000), las tendencias ideológicas de los individuos (Berry, 2006), la actuación de los medios de comunicación (Igartúa, Muñiz, Otero, Cheng \& Gómez, 2008) y el contexto nacional, en particular, el estado de la economía (Wilkes, Guppy \& Farris, 2008). Recientemente, Ward y Masgoret (2008), al probar la adecuación de un modelo de predicción de base psicosocial, en la relación de algunas variables con las actitudes hacia la inmigración y la política nacional de inmigración, pusieron de manifiesto que existen importantes indicios para creer que una fuerte ideología multicultural, altos niveles de contacto y bajos niveles de sentimientos de amenaza se relacionan, directamente, con actitudes positivas hacia los inmigrantes y que estas actitudes, a su vez, se relacionan positivamente con el respaldo a las políticas pro-inmigración. En esta misma línea de propuestas, la "Teoría del cosmopolitismo / provincianismo” (Chandler \& Tsai, 2001) postula que las personas con una visión más global del mundo muestran actitudes significativamente más favorables hacia la inmigración que aquellas personas que no presentan estas características. Para Haubert y Fusell (2006), las personas cosmopolitas se caracterizan por ser individuos muy educados, con ocupaciones de "cuello blanco", con una ideología política más liberal, que han vivido en el extranjero y que rechazan el etnocentrismo. Según estos autores, la visión cosmopolitan / parochial del mundo influye en las percepciones sobre las repercusiones sociales y económicas de la inmigración y éstas, a su vez, perfilan las actitudes de los sujetos.

Finalmente, el estudio de las actitudes resulta muy relevante para la comprensión de la conducta social humana por diversas razones, entre las cuales, Briñol, Falces y Becerra (2007) destacan la influencia que tienen sobre la forma en que piensan y actúan las personas y porque, además, reflejan la interiorización de los valores, de las normas y de las preferencias que rigen los grupos y las orga- 
nizaciones sociales, se considera que es importante conocer las actitudes que los estudiantes chilenos manifiestan hacia los inmigrantes. Además, se puede cuestionar si el sexo o si el hecho de tener experiencia de relación con personas de otras culturas introducen diferencias en tales actitudes. Y, como para conocer las actitudes es imprescindible utilizar las herramientas de evaluación adecuadas que permitan recoger una información fiable, analizar las propiedades del instrumento de evaluación constituye otro propósito prioritario para este estudio.

En suma, tres son los objetivos de esta investigación y que se concretan en los siguientes: en primer lugar, analizar las propiedades del instrumento de evaluación de las actitudes hacia la inmigración en el contexto universitario chileno; en segundo lugar, valorar el nivel de prejuicio manifiesto de los estudiantes chilenos hacia los inmigrantes, así como las dimensiones en las que se asienta, y, por último, analizar si el sexo o la experiencia de relación con personas de otras culturas introduce diferencias en las actitudes de los estudiantes chilenos, tal y como se ha puesto de manifiesto en otros contextos.

\section{Método}

\section{Participantes}

Participaron 117 estudiantes de psicología de tres universidades localizadas en la octava y en la novena región de Chile. La técnica de selección es de muestreo incidental (por una parte, universidades con las que se mantienen vínculos de docencia o de investigación y, por otra, la disponibilidad de los sujetos para responder al cuestionario). La mayoría son mujeres y los hombres representan el 34,2\% (77 mujeres y 40 hombres). El rango de edad oscila entre 18 y 33 años $(M=20,2 ; D T=$ 2,83).

\section{Variables e instrumento}

Los participantes cumplimentaron la Escala de Actitudes hacia la Inmigración, elaborada en la Universidad de Extremadura por León, Mira y Gómez (2007). El instrumento contiene 32 elementos que consisten en enunciados ante los que se ha de manifestar el grado de acuerdo, en una escala tipo Likert de 5 puntos que va desde Nada a Mucho. La fiabilidad de la escala, medida a través del coeficiente Alfa de Cronbach, es de 0,90 y está compuesta por cinco factores que, en su conjunto, explican el 52,5\% de la varianza. Estos factores o variables latentes son:

1) Principios y políticas de igualdad, que hace referencia a políticas de implantación de principios igualitarios y que expresa ideas como "estoy de acuerdo en que se proporcione a los inmigrantes asistencia sanitaria pública”, "estoy de acuerdo con una ley que permita cobrar el subsidio por desempleo a los inmigrantes" o "los inmigrantes que residen en nuestro país, deben tener derecho a votar en las elecciones”. Presenta una consistencia interna $\alpha=0,88$ y explica el $16,55 \%$ de la varianza.

2) Distancia social positiva, que explica el $12,65 \%$ de la varianza, tiene un coeficiente $\alpha=0,88$ y hace referencia a situaciones de relación de intimidad con inmigrantes que están relacionadas con expresiones como "no me importaría que un grupo de inmigrantes fuesen mis vecinos" o "me importaría tener un jefe que fuera inmigrante".

3) Distancia social negativa que se refiere a experiencias que no se compartirían con inmigrantes tales como "no iría a la piscina si hubiera inmigrantes" o "no compraría a vendedores ambulantes inmigrantes". Explica el 8,78\% de la varianza y su consistencia interna es $\alpha=0,73$.

4) Favorabilidad positiva que expresa ideas como "los inmigrantes son gente honesta" o "los inmigrantes son gente trabajadora” que están referidas a cualidades de los inmigrantes. Presenta un $\alpha$ de Cronbach de 0,73 y explica el 43,65\% de la varianza.

5) Favorabilidad negativa explica el 6,13\% de la varianza, muestra un $\alpha=0,65$ y hace referencia a aspectos negativos de los inmigrantes tales como "los inmigrantes son habitualmente unos irresponsables" o "los inmigrantes son de poco fiar". 


\section{Procedimiento}

La aplicación del instrumento fue realizada por un encuestador, previamente instruido, quien informó a los estudiantes sobre las características de la investigación y les solicitó su colaboración, destacando que la participación era voluntaria y anónima. Tras obtener su consentimiento verbal, en una sesión, en su aula habitual, los participantes tuvieron un tiempo indefinido para completar la encuesta, contaron con la asistencia del encuestador para aclarar sus dudas y no recibieron retribución. Se siguieron los criterios de la Comisión Nacional de Investigación Científica y Tecnológica de Chile, en lo referido a la confidencialidad de la información obtenida.

\section{Diseño y análisis de los datos}

Se trabajó con un diseño correlacional básico de tipo descriptivo-exploratorio. Para analizar la escala, se realizaron análisis de fiabilidad (empleando el coeficiente Alfa de Cronbach), análisis de discriminación (tomando como índice el coeficiente de correlación de Pearson entre la puntuación del ítem y la puntuación total de la escala) y análisis factoriales (empleando el método de extracción de componentes principales y de rotación Varimax con Kaiser). Para analizar las actitudes, se obtuvieron medidas de tendencia central, variabilidad, asimetría y curtosis y se realizaron contrastes de medias utilizando la prueba $t$ de Student. Para realizar estos análisis se empleó el programa SPSS (versión 15.0)

\section{Resultados}

\section{Análisis de la escala}

Como el instrumento de evaluación fue construido en un contexto (Extremadura) muy diferente al de aplicación (VIII y IX regiones de Chile), las respuestas a la Escala de Actitudes hacia la Inmigración (León et al., 2007) fueron sometidas a análisis descriptivos, de fiabilidad y de discriminación de los elementos. Los resultados señalan que las variables se ajustan a la distribución normal, que aportan fiabilidad a la escala y que presentan una adecuada capacidad de discriminación. Sin embargo, también indican que los elementos 8, 17, 31 y 32 no favorecen la consistencia interna del instrumento. Por tal motivo, fueron eliminados.

Por otra parte, considerando las respuestas de los sujetos a la escala como variables directamente observadas, se llevó a cabo con las mismas un análisis factorial. Se empleó el método de componentes principales con rotación Varimax con Kaiser. La rotación convergió tras 6 iteraciones. La medida de adecuación muestral era aceptable $(K M O=0,749)$ y la prueba de esfericidad de Bartlett resultó estadísticamente significativa $\left(\chi^{2}=1349 ; g l=378 ; p<0,001\right)$. Ambos valores indicaron que era adecuado realizar el análisis factorial del instrumento y que se podía rechazar la hipótesis de que la matriz de correlaciones era nula.

Del análisis factorial resultaron cinco factores que, en su conjunto, explicaron el 54,11\% de la varianza de la escala (el elemento 21 no presentó saturación superior a 0,30 en ningún factor).

En el primer factor saturaron los elementos 1, 2, 3, 4, 5, 6 y 7 y se refiere a aspectos positivos 0 negativos que se asocian a la personalidad de los inmigrantes (honestos, trabajadores, irresponsables, etc.). Dada la semejanza de éste factor con el obtenido por León et al. (2007), lo denominamos Favorabilidad. Explica el 22,93\% de la varianza y el coeficiente Alfa de Cronbach era de 0,83.

Al segundo factor, por su completa similitud con el factor I del instrumento original, lo llamamos Principios y políticas de igualdad, explicó el 9,58\% de la varianza y el índice de fiabilidad fue de 0,79. Saturaron en él los elementos 22, 23, 24, 25, 26, 27, 28, 29 y 30 y hace referencia a derechos fundamentales y a políticas de igualdad de oportunidades (derecho a vivienda, derecho a educación, derecho a atención sanitaria o derecho a subsidios).

El tercer factor se refiere a situaciones que no se compartirían con personas inmigrantes (no iría a la piscina si hubiera inmigrantes, no iría al cine o no compraría a vendedores inmigrantes), agrupa 
a los elementos 9, 10, 11, 12, y 13. Por su similitud con el factor III obtenido por León et al. (2007), lo denominamos Distancia social negativa. Explicó el 8,28\% de la varianza y la consistencia interna fue de 0,81 .

El cuarto factor agrupa a los elementos 14, 18 y 20 y se relaciona con situaciones de proximidad con personas inmigrantes (tener un compañero de trabajo, un médico o vecinos inmigrantes). Por su semejanza con el factor II de la escala original, lo llamamos Distancia social positiva. Explicó el 6,88\% de la varianza de la escala y el coeficiente Alfa de Cronbach fue de 0,71.

El quinto factor explicó el 6,43\% de la varianza, el índice de fiabilidad fue de 0,71 y lo denominamos Aceptabilidad. Hace referencia a relaciones interpersonales íntimas que se mantendrían con personas inmigrantes (me casaría, mantendría relaciones sexuales o aceptaría tener como compañero de habitación de un hospital a un inmigrante).

Por su parte, el análisis de discriminación por factores, señaló valores correlacionales que oscilaron entre 0,35 y 0,73, es decir, los factores presentaron una adecuada capacidad de discriminación.

En síntesis, la escala quedó compuesta por 27 elementos que se agruparon en cinco factores, los índices de fiabilidad de éstos fluctuaron entre 0,71 y 0,83, y presentaron una aceptable capacidad discriminativa. Estás características difieren levemente de las obtenidas por León et al. (2007), quienes presentan una escala compuesta por 32 elementos, que se agrupan en cinco factores que explican el 52,5\% de la varianza, con índices de fiabilidad que oscilan entre 0,65 y 0,88. Tales discrepancias pueden justificarse en función de las diferencias, tal vez culturales, tal vez sociales, entre las poblaciones de las que provienen los participantes de sendas investigaciones.

Análisis descriptivos de las actitudes hacia la inmigración

Por adición de las puntuaciones de los elementos que componen cada factor, se generan las variables latentes favorabilidad, principios y políticas de igualdad, distancia social negativa, distancia social positiva y aceptabilidad. El análisis descriptivo de éstas variables (ver la Tabla 1), señaló que los valores de asimetría y de curtosis están comprendidos entre $-1,96$ y $+1,96$, es decir, que se ajustan razonablemente a la distribución normal, excepto el valor de curtosis de la variable distancia social positiva $(2,75)$.

Tabla 1 - Análisis descriptivo de las actitudes hacia la inmigración

\begin{tabular}{lccccc}
\hline Variable & M & DT & Varianza & Asimetría & Curtosis \\
\hline Distancia social negativa & 1,86 & 3,15 & 9,91 & 1,88 & 2,75 \\
Favorabilidad & 8,18 & 4,62 & 21,34 & $-0,09$ & $-1,26$ \\
Principios y políticas de igualdad & 10,49 & 6,23 & 38,75 & 0,13 & $-0,95$ \\
Aceptabilidad & 4,20 & 2,56 & 6,57 & 0,06 & $-0,09$ \\
Distancia social positiva & 7,55 & 3,81 & 14,54 & $-0,55$ & $-0,72$ \\
\hline
\end{tabular}

Las bajas puntuaciones en la mencionadas variables indican actitudes positivas y, al contrario, puntuaciones elevadas significan actitudes negativas. En la tabla II se presenta un resumen del análisis de frecuencias en función de que las actitudes sean positivas, intermedias y negativas. Los resultados, en términos generales, indican que los sujetos manifiestan una actitud favorable hacia la inmigración. En distancia social negativa, puntuaciones entre 0 y 6 reflejan una actitud positiva y entre 14 y 20 negativa. Así, la gran mayoría de los individuos (el 90,6\%) muestra puntuaciones que indican una tendencia positiva para compartir espacios públicos y para establecer relaciones esporádicas con los inmigrantes, manifestando que pondría en marcha conductas como ir al cine o mantener cierto grado de amistad con personas inmigrantes. En favorabilidad, puntuaciones entre 0 y 9 indican actitudes positivas y entre 19 y 28 actitudes negativas. Se puede observar que la mayoría 
de los participantes (el 63,2\%) puntúa en el sentido de considerar que los inmigrantes son personas honestas, responsables, tolerantes e inteligentes. En principios y políticas de igualdad, las actitudes positivas se expresan con puntuaciones entre 0 y 11 y las negativas con puntuaciones entre 24 y 36 . Se observa que el $60,7 \%$ de los sujetos manifiesta una actitud que consiste en entender que los inmigrantes tienen derecho a la educación, a la atención sanitaria y a la vivienda que ofrece el país. En aceptabilidad, puntuaciones entre 0 y 3 significan actitud positiva y puntuaciones entre 9 y 12 indican actitud negativa. Casi la mitad de los sujetos (el 53\%) muestra una actitud favorable para establecer relaciones interpersonales íntimas con los inmigrantes. En distancia social positiva, la actitud positiva está representada por puntuaciones entre 0 y 3 y la negativa por puntuaciones entre 9 y 12. Es decir, casi la cuarta parte de los sujetos (el 23\%) muestra una actitud que se traduce en querer compartir espacios laborales o de residencia o buscar la atención profesional de un inmigrante, a la vez que el 38,5\% de los sujetos muestra actitudes negativas en este sentido.

Tabla 2 - Análisis de frecuencias de las actitudes hacia la inmigración

\begin{tabular}{|c|c|c|c|c|c|c|}
\hline \multirow{2}{*}{ Variable } & \multicolumn{2}{|c|}{ Actitud positiva } & \multicolumn{2}{|c|}{$\begin{array}{c}\text { Actitud } \\
\text { intermedia }\end{array}$} & \multicolumn{2}{|c|}{$\begin{array}{c}\text { Actitud } \\
\text { negativa }\end{array}$} \\
\hline & $f$ & $P$ & $f$ & $P$ & $f$ & $P$ \\
\hline & \multicolumn{2}{|c|}{$(0-6)$} & \multicolumn{2}{|c|}{$(7-13)$} & \multicolumn{2}{|c|}{$(14-20)$} \\
\hline Distancia social negativa & \multicolumn{2}{|c|}{$\begin{array}{r}106 \quad 90,6 \\
(0-9)\end{array}$} & \multicolumn{2}{|c|}{$\begin{array}{rr}10 & 8,5 \\
(10-18)\end{array}$} & \multicolumn{2}{|c|}{$\begin{array}{lr}1 & 0,09 \\
(19 & -28)\end{array}$} \\
\hline Favorabilidad & \multicolumn{2}{|c|}{$\begin{array}{r}74 \quad 63,2 \\
(0-11)\end{array}$} & \multicolumn{2}{|c|}{$\begin{array}{rr}43 & 36,8 \\
(12-23)\end{array}$} & \multicolumn{2}{|c|}{$\begin{array}{lr}0 & 0 \\
(24-36)\end{array}$} \\
\hline Principios y políticas de igualdad & \multicolumn{2}{|c|}{$\begin{array}{rr}71 \quad 60,7 \\
(0-3)\end{array}$} & \multicolumn{2}{|c|}{$\begin{array}{cc}46 & 39,3 \\
& (4-8)\end{array}$} & \multicolumn{2}{|c|}{$\begin{array}{lr}0 & 0 \\
(9-12)\end{array}$} \\
\hline Aceptabilidad & \multicolumn{2}{|c|}{$\begin{array}{c}62 \quad 53 \\
(0-3)\end{array}$} & \multicolumn{2}{|c|}{$\begin{array}{r}47 \quad 40,2 \\
\quad(4-8)\end{array}$} & \multicolumn{2}{|c|}{$\begin{array}{lr}8 & 6,8 \\
(9 & -12)\end{array}$} \\
\hline Distancia social positiva & & 23 & 45 & 38,5 & & 38,5 \\
\hline
\end{tabular}

Nota. $f=$ Frecuencia; $P$ = Porcentaje.

\section{Análisis diferenciales}

Las variables latentes se consideran variables agrupadas y son sometidas a análisis diferenciales en función del sexo y de la experiencia de relación con personas inmigrantes como variables dicotómicas de agrupamiento. Para ello se emplea la prueba $t$ de Student para muestras independientes. Los resultados de la prueba de Levene (ver la Tabla 3), indicaron que no existen diferencias estadísticamente significativas en las varianzas de las variables analizadas y esto permite asumir su homogeneidad. 
Tabla 3 - Prueba de Levene para la homogeneidad de las varianzas. Sexo y experiencia de relación con inmigrantes como variables de agrupamiento y actitudes hacia la inmigración como variables agrupadas

\begin{tabular}{lcc}
\hline Variable & $\mathrm{F}$ & $\mathrm{p}$ \\
\hline Sexo & & \\
Distancia social negativa & 0,880 & 0,350 \\
Favorabilidad & 0,011 & 0,916 \\
Principios y políticas de igualdad & 0,048 & 0,826 \\
Aceptabilidad & 0,280 & 0,598 \\
Distancia social positiva & 1,069 & 0,303 \\
Experiencia de relación & & \\
Distancia social negativa & 0,163 & 0,687 \\
Favorabilidad & 0,072 & 0,789 \\
Principios y políticas de igualdad & 1,810 & 0,181 \\
Aceptabilidad & 0,297 & 0,587 \\
Distancia social positiva & 1,616 & 0,434 \\
\hline
\end{tabular}

En relación con el sexo, según se observa en la Tabla 4, los resultados indican que existen diferencias estadísticamente significativas en las variables principios y políticas de igualdad $\left(t_{(115)}=\right.$ $-2,75 ; p=0,007)$ y aceptabilidad $\left(t_{(115)}=-2,09 ; p=0,039\right)$. Hay que tener en cuenta que, cuanto más bajo sea el valor medio $(M)$, más positiva es la actitud. De modo que las mujeres en relación con los hombres manifiestan una mejor actitud hacia los derechos básicos y hacia las políticas de igualdad de oportunidades para los inmigrantes (derecho a asistencia sanitaria, derecho a educación, derecho a vivienda, derecho a subsidios, derecho a voto). Además, también las mujeres muestran una actitud más positiva para establecer relaciones interpersonales íntimas con las personas inmigrantes en comparación con los hombres.

Tabla 4 - Prueba t de Student para el contraste de medias. Sexo como variable de agrupamiento y actitudes hacia la inmigración como variables agrupadas

\begin{tabular}{|c|c|c|c|c|c|c|}
\hline \multirow{2}{*}{ Variable } & \multicolumn{3}{|c|}{ Descriptivo } & \multicolumn{3}{|c|}{ Prueba $t$} \\
\hline & Sexo & $\mathrm{n}$ & M & $\mathrm{t}$ & gl & $\mathrm{p}$ \\
\hline \multirow{2}{*}{ Favorabilidad } & $\mathrm{M}$ & 77 & 7,78 & \multirow{2}{*}{$-1,30$} & \multirow{2}{*}{115} & \multirow{2}{*}{0,195} \\
\hline & $\mathrm{H}$ & 40 & 8,95 & & & \\
\hline \multirow{2}{*}{ Principios y políticas de igualdad } & M & 77 & 9,38 & \multirow{2}{*}{$-2,75$} & \multirow{2}{*}{115} & \multirow{2}{*}{$0,007 *$} \\
\hline & $\mathrm{H}$ & 40 & 12,63 & & & \\
\hline \multirow{2}{*}{ Distancia social negativa } & $\mathrm{M}$ & 77 & 1,66 & \multirow{2}{*}{$-0,96$} & \multirow{2}{*}{115} & \multirow{2}{*}{0,340} \\
\hline & $\mathrm{H}$ & 40 & 2,25 & & & \\
\hline \multirow{2}{*}{ Distancia social positiva } & M & 77 & 7,87 & \multirow{2}{*}{1,28} & \multirow{2}{*}{115} & \multirow{2}{*}{0,205} \\
\hline & $\mathrm{H}$ & 40 & 6,93 & & & \\
\hline \multirow{2}{*}{ Aceptabilidad } & M & 77 & 3,84 & \multirow{2}{*}{$-2,09$} & \multirow{2}{*}{115} & \multirow{2}{*}{$0,039 *$} \\
\hline & $\mathrm{H}$ & 40 & 3,88 & & & \\
\hline
\end{tabular}

$* p<0,05$ 
En la Tabla 5 se presentan los resultados de los análisis diferenciales en función de la experiencia de relación con personas inmigrantes. Éstos indicaron que existen diferencias significativas en las variables favorabilidad $\left(t_{(115)}=2,86 ; p=0,005\right)$ y principios y políticas de igualdad $\left(t_{(115)}=2,20\right.$; $p=0,030)$. En consecuencia, se deduce que los sujetos que se han relacionado con inmigrantes manifiestan una mejor percepción hacia las características de personalidad de estas personas (son honestos, responsables, trabajadores, tolerantes) y una actitud positiva hacia las iniciativas públicas que les favorezcan, en comparación con los individuos que no se han relacionado con inmigrantes.

Tabla 5 - Prueba t de Student para el contraste de medias. Experiencia de relación con inmigrantes como variable de agrupamiento y actitudes hacia la inmigración como variables agrupadas

\begin{tabular}{|c|c|c|c|c|c|c|}
\hline \multirow{2}{*}{ Variable } & \multicolumn{3}{|l|}{ Descriptivo } & \multicolumn{3}{|c|}{ Prueba $t$} \\
\hline & Relación & $n$ & $M$ & $t$ & $g l$ & $p$ \\
\hline \multirow{2}{*}{ Favorabilidad } & No & 44 & 9,70 & \multirow{2}{*}{2,86} & \multirow{2}{*}{115} & \multirow{2}{*}{$0,005 *$} \\
\hline & Sí & 73 & 7,26 & & & \\
\hline \multirow{2}{*}{ Principios y políticas de igualdad } & No & 44 & 12,09 & \multirow{2}{*}{2,20} & \multirow{2}{*}{115} & \multirow{2}{*}{$0,030^{*}$} \\
\hline & Sí & 73 & 9,52 & & & \\
\hline \multirow{2}{*}{ Distancia social negativa } & No & 44 & 2,11 & \multirow{2}{*}{0,67} & \multirow{2}{*}{115} & \multirow{2}{*}{0,507} \\
\hline & Sí & 73 & 1,71 & & & \\
\hline \multirow{2}{*}{ Distancia social positiva } & No & 44 & 8,05 & \multirow{2}{*}{1,10} & \multirow{2}{*}{115} & \multirow{2}{*}{0,274} \\
\hline & Sí & 73 & 7,25 & & & \\
\hline \multirow{2}{*}{ Aceptabilidad } & No & 44 & 4,23 & \multirow{2}{*}{0,10} & \multirow{2}{*}{115} & \multirow{2}{*}{0,920} \\
\hline & Sí & 73 & 4,18 & & & \\
\hline
\end{tabular}

$* p<0,05$

\section{Discusión}

La Escala de Actitudes hacia la Inmigración (León et al., 2007), aplicada a estudiantes universitarios chilenos, presentó características adecuadas, es decir, los cinco factores extraídos muestran una fiabilidad o consistencia interna y una capacidad de discriminación aceptables. Estas características difieren levemente de las obtenidas por León et al. (2007), lo cual nos parece razonable considerando que los participantes de éste estudio pertenecen a un contexto cultural distinto, en donde la inmigración y las variables sociales que forman parte de estos fenómenos, entendidas como las normas y leyes adoptadas por una determinada sociedad, así como las relaciones históricas entre los diversos grupos (Molero, Cuadrado \& Navas, 2003; Rodríguez, Betancor, \& Delgado, 2009) presentan sus propias características.

Teniendo en cuenta los resultados obtenidos, se pone de manifiesto que los participantes muestran bajos niveles de prejuicio manifiesto, aunque debemos considerar la influencia de las percepciones acerca de lo que se considera socialmente deseable que pudiesen afectar a los participantes ya que, según Morales (2003), pueden enmascarar actitudes latentes de mayor tendencia xenófoba. Por otra parte, alrededor de un tercio de las respuestas obtenidas, manifiestan una actitud intermedia y esto podría relacionarse con esa forma enmascarada e indirecta de hostilidad hacia el exogrupo (Dovidio \& Gaertner, 1991, 1998; García, Navas, Cuadrado \& Molero, 2003; McConahay, Hardee \& Batts, 1981; Navas, 1998; Pettigrew \& Meertens, 1995; Rueda, Navas \& Gómez, 1995) que, en este caso en particular, se caracterizaría, principalmente, por una tendencia a evitar las relaciones íntimas con los inmigrantes, limitando las interacciones a espacios públicos, y por una tendencia negativa a compartir espacios laborales o de residencia. No obstante, es necesario precisar que estos hallazgos no pueden ser generalizados a toda la población chilena, ya que los sujetos de este estudio poseen un nivel educacional distinto al general. 
En cuanto a las explicaciones para las diferencias encontradas en las variables principios y políticas de igualdad y aceptabilidad entre hombres y mujeres, hay que buscarlas en variables psicológicas, como las características de personalidad o en variables sociales, como la calidad de las relaciones mantenidas con personas de grupos culturales distintos al propio, o en variables cognitivas, como el tipo de información que se tenga sobre el fenómeno de la inmigración y la interpretación que se realice de ésta. Por su parte, las diferencias que se observan en las variables favorabilidad y principios y políticas de igualdad, que favorecen a quienes se han relacionado con inmigrantes, concuerdan con la hipótesis que plantea que un mayor contacto con el exogrupo, favorece el desarrollo de actitudes más positivas hacia sus miembros (Allport, 1954; Kalin y Berry, 1982; Pettigrew y Tropp, 2000).

Hay que destacar que, si bien los análisis realizados nos sugieren que la Escala de actitudes hacia la Inmigración (León et al., 2007) es un instrumento fiable para abordar el estudio de las actitudes en el contexto chileno, sería conveniente estudiar el comportamiento del instrumento en una muestra más amplia y en la que estuvieran representados los diferentes niveles educativos de la población chilena. Por otra parte, los resultados obtenidos aportan información valiosa sobre la actitud de los chilenos que merecen ser analizadas con mayor profundidad y también en una muestra más amplia de individuos que abarcara a las distintas regiones del país. Estos aspectos pueden considerarse autocríticas y, al mismo tiempo, deficiencias a superar en trabajos futuros.

Igualmente sería interesante poder valorar las respuestas considerando algunas de las variables mencionadas anteriormente y analizar si la ideología política de los participantes, si su visión del mundo (global o local) o si la percepción subjetiva de bonanza o estabilidad económica del país introducen diferencias en sus actitudes hacia la inmigración, así como, también, diferenciar las actitudes dirigidas a distintos grupos culturales (peruanos, argentinos, colombianos, etc.). Para finalizar, como implicación práctica derivada de los resultados, cabe plantearse la posibilidad de llevar a cabo un programa para el cambio de actitudes en los sujetos porque, en distancia social positiva, más de un tercio de los participantes manifiesta una actitud negativa y, en todas las variables (excepto en distancia social negativa), los porcentajes de sujetos con puntuaciones intermedias parecen indicar la existencia del prejuicio sutil.

\section{Referencias}

Allport, G. W. (1954). The nature of prejudice. Reading, M.A., EE.UU.: Adisson-Wesley.

Arkin, H., \& Colton, R. (1962). Tables for statiscians. Nueva York, EE.UU.: Barnes \& Noble.

Berry, J. (2006). Mutual attitudes among immigrants and ethnocultural groups in plural societies. International Journal of Intercultural Relations, 30, 719-734.

Briñol, P., Falces, C., \& Becerra, A. (2007). Actitudes. En J. F. Morales, M. Moya, E. Gaviria \& I. Cuadrado (Coords.), Psicología social (pp. 457-490). Madrid, España: McGraw-Hill.

Chandler, C. R., \& Tsai, H. M. (2001). Social factors influencing immigration attitudes: An analysis of data from the general social survey. The Social Science Journal, 38, 277-288.

Dovidio, J., \& Gaertner, S. (1991). Changes in the expression and assessment of racial prejudice. En H. Knopke, R. Norrel \& R. Rogers (Eds.), Opening doors:Perspectives on race relations in contemporary America (pp.119-448). Tuscaloosa, EE.UU.: The University of Alabama.

Dovidio, J., \& Gaertner, S. (1998). The causes, consequences, and challenges of aversive racism. En J. Eberhardt \& S. Fiske (Eds.), Confronting racism: The problem and the response (pp. 3-32). Londres, Inglaterra: Sage.

Dustmann, C., \& Preston, I. (2004). Racial and economic factors in attitudes to immigration. Centre for Research and Analysis of Migration (CreAM), Department of Economics. Londres, Inglaterra: University College London. 
Gang, I. N., Rivera-Batiz, F. L., \& Yun, M. S. (2001). Economic strain, ethnic concentration and attitudes towards foreigners in the European Union. Working Paper 200214. Nueva Brunswick, N.J., EE.UU.: Rutgers University, Department of Economics.

García, M. C., Navas, M. S., Cuadrado, I., \& Molero, F. (2003). Inmigración y prejuicio: Actitudes de una muestra de adolescentes almerienses. Acción Psicológica, 2 (2), 137-147.

Gutiérrez, D. (1995). Walls and morrors: mexican americans, mexican immigrants and the politic of ethnicity. Berkeley, C.A., EE.UU.: University of California Press.

Haubert, J., \& Fusell, E. (2006). Explaining pro-immigrant sentiment in the U. S.: Social class, cosmopolitanism, and perceptions of immigrants. International Migration Review, 40, 489507.

Igartúa, J., Muñiz, C., Otero, J., Cheng, L., \& Gómez, J. (2008). Recepción e impacto socio-cognitivo de las noticias sobre inmigración. Revista de Psicología Social, 23 (1), 3-16.

Kalin, R., \& Berry, J. (1982). The social ecology of ethnic attitudes in Canadian. Canadian Journal of Behavioral Science, 25, 28-44.

Katz, I., \& Hass, R. C. (1988). Racial ambivalence and American value conflict: Correlational and priming studies of dual cognitive structure. Journal of Personality and Social Psychology, 44, 893-905.

León, B., Mira, A., \& Gómez, T. (2007). Evaluación de las opiniones sobre la inmigración y la multiculturalidad en la escuela de alumnos de magisterio. Revista Electrónica de Investigación Psicoeducativa, 5 (2), 259-282.

McConahay, J. B., Hardee, B. B., \& Batts, V. (1981). Has racism declined in America? It depends upon who is asking and what is asked. Journal of Conflict Resolution, 25, 563-579.

Molero, F., Cuadrado, I., \& Navas, M. (2003). Las nuevas expresiones del prejuicio racial: Aspectos teóricos y empíricos. En J. F. Morales \& C. Huici (Coords.), Estudios de Psicología Social (pp. 83-117). Madrid, España: UNED.

Molero, F., Navas, M. S., \& Morales, J. F. (2001). Inmigración, prejuicio y exclusión social: reflexiones en torno a algunos datos de la realidad española. International Journal of Psychology and Psychological Therapy, 1 (1), 11-32.

Morales, J. F. (2003). El estudio de la exclusión social en la psicología social. En J. F. Morales \& C. Huici (Coords.), Estudios de psicología social (pp. 509-538). Madrid, España: UNED.

Morales, J. F., \& Huici, C. (1999). Grupos. En J. F. Morales (Coord.), Psicología social (pp. 685700). Madrid, España: McGraw-Hill.

Navas, M. (1998). Nuevos instrumentos de medida para el nuevo racismo. Revista de Psicología Social, 13 (2), 233-239.

Olzak, S. (1992). The dynamic of ethnic competition and conflict. Stanford, C.A., EE.UU.: Stanford University Press.

O’Rourke, K., \& Sinnott, R. (2006). The determinants of individual attitudes towards immigration. European Journal of Political Economy, 22, 838-861.

Pantoja, A. (2006). Against the tide? Core american values and attitudes toward US immigration policy in the mid-1990s. Journal of Ethnic and Migration Studies, 32, 515-531.

Palmer, D. (1996). Determinants of canadian attitudes towards immigration: More than just racism? Canadian Journal of Behavioural Science, 28, 180-192.

Pettigrew, T., \& Meertens, R. W. (1995). Subtle and blantant prejudice in Western Europe. European Journal of Social Psychology, 25, 57-75. 
Pettigrew, T., \& Tropp, L. (2000). Does intergroup contac reduce prejudice: Recent meta-analytic findings. En S. Oskam (Ed.), Reducing Prejudice and Discrimination (pp. 93-114). Mahwah, N.J., EE.UU.: Lawrence Erlbaum.

Quesille, A. (2007). Inmigrantes, refugiados y derechos humanos. En N. Espejo (Coord.), Informe Anual sobre Derechos Humanos en Chile. Hechos 2006 (pp. 317-362). Santiago de Chile: Universidad Diego Portales.

Raijman, R., Semyonov, M., \& Schmidt, P. (2003). Do foreigners deserve rights? Determinants of Public Views Towards Foreigners in Germany and Israel. European Sociological Review, 4, 379-392.

Rodríguez, A., Betancor, V., \& Delgado, N. (2009). La norma social sobre la expresión de prejuicio explícito hacia diferentes grupos sociales. Revista de Psicología Social, 24 (1), 17-20.

Rueda, J. F., Navas, M. S., \& Gómez, C. (1995). Las nuevas expresiones del racismo: adaptación de una escala de prejuicio sutil. En J. C. Sánchez \& A. M. Ullán (Comps.), Procesos psicosociales básicos y grupales (pp. 357-370). Salamanca, España: Eudema.

Semyonov, M., Raijman, R., Yom Tov, A., \& Schmidt, P. (2004). Population size, perceived threat, and exclusion: A multiple-indicators analysis of attitudes toward foreigners in Germany. Social Science Research, 33, 681-701.

Simon, R. J., \& Sikich, K. W. (2007). Public attitudes toward immigrants and immigration policies across seven nations. International Migration Review, 41 (4), 956-562.

Tajfel, H. (1981). Human groups and social categories. Cambridge, Inglaterra: Cambridge University Press.

Tucci, I. (2005). Explaining attitudes towards immigration: New pieces to the puzzle. Discussion Paper 484. Berlin Alemania: German Institute for Economic Research.

Turner, J., Hogg, M., Oakes, P., Reicher, S., \& Wetherell, M. (1987). Rediscovering the social group: A self-categorisation theory. Oxford, Inglaterra: Blackwell.

Ward, C., \& Masgoret, A. (2008). Attitudes toward immigrants, immigration, and multiculturalism in New Zealand: A social psychological analysis. International Migration Review, 42 (1), 227248.

Wilkes, R., Guppy, N., \& Farris, L. (2008). "No thanks, we're full”: Individual characteristic, national context, and changing attitudes toward immigration. International Migration Review, 42 (2), 302-329. 